\title{
Tasy e o ensino em saúde
}

\author{
Luís Felipe Pissaia \\ Mestre e Doutorando em Ensino \\ Especialista em Gestão e Auditoria dos Serviços da Saúde \\ Universidade do Vale do Taquari - Univates - Brasil \\ $\bowtie$ lpissaia@universo.univates.br \\ Márcia Jussara Hepp Rehfeldt \\ Doutora em Informática na Educação \\ Universidade do Vale do Taquari - Univates - Brasil \\ Arlete Eli Kunz da Costa \\ Doutora em Ambiente e Desenvolvimento \\ Universidade do Vale do Taquari - Univates - Brasil \\ Claudete Moreschi \\ Doutora em Ambiente e Desenvolvimento \\ Universidade Regional Integrada do Alto Uruguai e das Missões - Brasil
}

Recebido em 6 de agosto de 2020

Aceito em 16 de novembro de 2021

\section{Resumo:}

Este estudo objetiva descrever as contribuições do software Tasy no estabelecimento da relação entre teoria e prática nas disciplinas de Enfermagem na Saúde do Adulto e Idoso I e II de um curso de Enfermagem. Em suma, trata-se de uma pesquisa qualitativa, descritiva e exploratória, a qual teve como participantes trinta e um estudantes do curso de Enfermagem da Universidade do Vale do Taquari. Todos os participantes estavam matriculados nas disciplinas destacadas, durante o primeiro semestre de 2018. 0 instrumento de levantamento de informações deste estudo foi o diário de campo do pesquisador que esteve presente durante as 20 horas aula da pesquisa, sendo que todos os achados foram reunidos em pontos focais compatíveis e categorizados com aproximações da Análise de Conteúdo. Os resultados evidenciaram que os participantes consideram o Tasy como um facilitador na realização da Sistematização da Assistência de Enfermagem. Eles destacam suas funcionalidades, como a de prontuário, do registro de informações e, por consequente, qualificador da assistência oferecida ao cliente. Pontua-se nos achados, a facilidade em utilizar o software na aplicação da assistência de enfermagem, reforçando a necessidade em tê-lo nos diferentes serviços de saúde. Observou-se também que o software possibilitou a realização eficaz e integral da Sistematização da Assistência de Enfermagem, estimulando a construção entre a teoria e a prática. Deste modo, também foram pontuados a eficácia do software, enquanto modelo de gestão em saúde e ferramenta eficaz para a comunicação entre as equipes.

Palavras-chave: Ensino em Saúde. Ensino em Enfermagem, Teoria e Prática, Ferramentas de Ensino, Tasy, Sistematização da Assistência de Enfermagem.

\section{Tasy and health education}

\begin{abstract}
:
This study aims to describe the contributions of the Tasy software in the set up of the relationship between theory and practice in the disciplines of Nursing in Adult and Elderly Health I and II of a Nursing course. In short, this is a qualitative, descriptive and exploratory research, which had as
\end{abstract}


participants thirty-one students of the Nursing course of the University of Vale do Taquari. All participants were enrolled in the above mentioned subjects during the first semester of 2018. The information gathering instrument of this study was the field diary of the researcher who was present during the 20 hours class of the research. All the findings were gathered in compatible focal points and categorized whits Content Analysis approaches. The results showed that the participants consider Tasy as a facilitator in the carry out of the Systematization of Nursing Assistance. They highlight their functionalities, such as the medical record, the information record and, therefore, qualifier of the helpoffered to the client. The findings are based on the ease of using the software in the apply of nursing care, reinforcing the need to have it in the different health services. It was also observed that the software made possible the effective and integral realization of the Systematization of Nursing Assistance, stimulating the construction between theory and practice. In this way, the effectiveness of the software was also evaluated as a health management model and an effective tool for communication between teams.

Keywords: Teaching in Health, Teaching in Nursing, Theory and practice, Teaching Tools, Tasy, Systematization of nursing care.

\section{Tasy y educación sanitaria}

\section{Resumen:}

Este estudio tiene como objetivo describir las contribuciones del software Tasy para establecer la relación entre la teoría y la práctica en los cursos de Enfermería en Salud de Adultos y Ancianos I y II de un curso de Enfermería. En resumen, se trata de una investigación cualitativa, descriptiva y exploratoria, que tuvo como participantes treinta y un estudiantes del curso de Enfermería en la Universidad de Vale do Taquari. Todos los participantes se inscribieron en las disciplinas destacadas, durante el primer semestre de 2018. El instrumento para recopilar información de este estudio fue el diario de campo del investigador que estuvo presente durante las 20 horas de la clase de investigación, y todos los hallazgos se recopilaron en puntos focales compatibles y categorizados con enfoques para el Análisis de Contenido. Los resultados mostraron que los participantes consideran a Tasy como un facilitador en la realización de la Sistematización del Cuidado de Enfermería. Destacan sus funcionalidades, como el registro médico, el registro de información y, en consecuencia, el calificador de la asistencia ofrecida al cliente. Puntuado en los hallazgos, la facilidad en el uso del software en la aplicación de cuidados de enfermería, lo que refuerza la necesidad de tenerlo en diferentes servicios de salud. También se observó que el software permitió la realización efectiva e integral de la Sistematización del Cuidado de Enfermería, estimulando la construcción entre la teoría y la práctica. De esta manera, también se calificó la efectividad del software, como un modelo de gestión de la salud y una herramienta eficaz para la comunicación entre los equipos.

Palabras clave: Docencia en Salud, Docencia en Enfermería, Teoría y práctica, Herramientas de enseñanza, Tasy, Sistematización de la atención de enfermería..

\section{INTRODUÇÃo}

O uso de tecnologias da informática na área da saúde faz parte de um limiar de mudanças em que os serviços prestadores de assistência médica necessitam oferecer um cuidado de qualidade para seus clientes, bem como gerenciar seus custos de maneira sustentável (PISSAIA et al., 2018). Sob este limiar de gerenciamento e ensino em saúde observa-se que nas últimas décadas, principalmente após os anos 1990 e com a virada dos anos 2000, tornou-se permanente a necessidade de qualificar a assistência por meio de uma 
aliança entre estes dois fatores (YAMAMOTO; BANDEIRA-PAIVA; ITO, 2015). Neste itinerário de qualificadores da assistência, destaca-se o uso de tecnologias em saúde, por meio de softwares, que conforme Pissaia et al. (2017), sintetizam a necessidade de integrar e flexibilizar os processos de ensino e gestão que ocorrem em serviços de saúde.

Acrescenta-se, também, a opinião de Silva, Gonçalves e Santos (2017), sobre a junção de tais características citadas, que ocorre por meio da utilização do software Tasy, ferramenta comercializada pela empresa Philips Health Care, que oferece um sistema por meio de plataforma virtual para soluções em saúde, personalizada a partir das demandas de cada serviço. Além disso, em seu site online, a Philips possui uma página específica para esta a oferta destas ferramentas, sendo que, em aba específica denominada "Solução de Gestão em Saúde".

Em resumo, na aba citada estão descritas as finalidades do software, elencando inicialmente sua indicação, propostas de adaptabilidade às diferentes demandas dos serviços de saúde, além dos indicativos de qualificação das atividades realizadas por meio da melhoria contínua do serviço. Dessa forma, segue o trecho abaixo exemplificando as informações:

Solução completa e 100\% integrada para a gestão de organizações de saúde públicas e privadas. Aderentes às mais variadas realidades de negócios, independente do seu porte e da complexidade dos seus processos, atendendo a: prestadores de serviços: hospital, clínica, banco de sangue, home care entre outros. Gerenciamento eficiente das atividades administrativas, financeiras, assistenciais e operacionais. Mais de 50 módulos que recebem melhorias contínuas por meio das versões disponibilizadas semanalmente. Relatórios e indicadores de gestão que fornecem o controle e o acompanhamento da performance operacional e financeira, apoiando a tomada de decisão (PHILIPS, 2018).

Utilizando-se destes pressupostos tecnológicos, durante a implantação de um Ambulatório de Especialidades Médicas, Pissaia e Beschorner (2016) realizaram um relato de experiência em que enfatizaram que o uso do Tasy fortaleceu principalmente o uso da metodologia de trabalho paperless, que delimita o uso de sistemas e soluções estritamente informatizadas para gestão em saúde, unificando as informações do prontuário do cliente. Neste caso, funcionando como um banco de dados para a assistência e ensino em saúde. Ainda, no site online da Philips, enfatizam-se os benefícios do sistema no contexto de um serviço de saúde, deixando clara a possibilidade de utilização do software como ferramenta de ensino, ao passo que, oferece a segurança e aporte para a tomada de decisões embasadas em um sistema 
de controle e integração dos dados gerados pelos processos assistenciais, conforme o trecho que segue:

\begin{abstract}
Integração, controle e otimização dos processos. Melhoria nos resultados financeiros, com gestão de suprimentos, faturamento, finanças e controladoria. Qualidade e segurança clínico-assistencial e melhoria no atendimento ao paciente com o uso da prescrição e do prontuário eletrônico, das ferramentas de gestão do atendimento e de suporte clínico à decisão. Segurança, rastreabilidade e integração das informaç̧ões. Diferenciação da empresa no seu mercado de atuação, em virtude dos benefícios proporcionados pela tecnologia. Integrado a vários produtos o portfólio de soluções Philips (PHILIPS, 2018).
\end{abstract}

Acrescenta-se também o grande incentivo do Tasy para o ensino, indicado em sua própria essência por Pissaia e Beschorner (2016), ao unificar e flexibilizar os processos que envolvem a assistência em saúde. Desde modo, torna-se evidente a construção de limiares entre o ensino teórico produzido em sala de aula e o prático desenvolvido por meio do software, já que ambos constroem-se durante a utilização pelos estudantes (PISSAIA; BESCHORNER, 2016).

Sendo assim, Silva, Gonçalves e Santos (2017) fundamentam o perfil gerenciador da ferramenta, incentivando a adoção de métodos tecnológicos para garantia da qualidade dos indicadores, tanto para a sustentabilidade dos negócios, quanto para a satisfação dos clientes atendidos, por meio de processos claros e evidentes cientificamente. Pissaia et al. (2017) acrescentam a necessidade de conhecimento dos profissionais para manusearem o software, ficando explícitas as recomendações sobre as vivências práticas na utilização de tecnologias.

Sendo assim, o objetivo deste estudo é descrever as contribuições do software Tasy no estabelecimento da relação entre teoria e prática entre as disciplinas de "Enfermagem na Saúde do Adulto e Idoso" I e II de um curso de Enfermagem.

\title{
METODOLOGIA
}

Trata-se de uma pesquisa qualitativa, descritiva e exploratória. Deste modo, utilizouse de preceitos imparciais para a sua realização, consonante com a necessidade de estimular a representação de todos os fatos sem interferência externa, conforme previsto por Moreira (2011). 
Para a realização desta pesquisa participaram trinta e um estudantes, com média de 24 anos de idade, matriculados nas disciplinas de Enfermagem na Saúde do Adulto e Idoso I e II do curso de Enfermagem da Universidade do Vale do Taquari. A coleta de dados ocorreu no primeiro semestre de 2018, nas salas de aula da Universidade do Vale do Taquari e nas dependências do Centro Clínico Univates, serviço escola da mesma instituição que abriga alguns estágios e práticas dos cursos de graduação e técnicos.

Neste estudo utilizou-se como instrumento o diário de campo do pesquisador, o qual esteve presente durante as 20 horas de aula acompanhadas nas referidas disciplinas. Por meio de excertos deste registro pretende-se dissertar sobre o objetivo proposto, sendo que os achados foram transcritos em sua íntegra e reunidos em pontos focais compatíveis que originaram as categorias temática de análise, conforme aproximações da Análise de Conteúdo, de Bardin (2016).

Para a realização das discussões, foram omitidos os nomes dos participantes, sendo utilizados codinomes representados pelo prefixo " $Q$ " seguido de números ordinais sorteados aleatoriamente. Desta forma, foram seguidos os preceitos éticos informados pela Resolução 466/12 do Conselho Nacional de Saúde, a qual direciona os princípios para a realização de pesquisas com seres humanos (BRASIL, 2012). Destaca-se também que a realização da pesquisa esteve condicionada à aprovação da Universidade do Vale do Taquari e do Centro Clínico Univates, ambos por meio de Carta de Anuência.

\section{RESULTADOS E DISCUSSÃo}

Nesta seção apresentam-se os achados e discussões do presente estudo. Os resultados foram categorizados por meio de seus pontos focais compatíveis, representando assim uma melhor compreensão sobre os achados. Na primeira categoria, denominada "Tasy e as contribuições no estabelecimento da relação entre teoria e prática" disserta-se sobre a utilização do Tasy enquanto facilitador da relação entre teoria e prática. Já na segunda categoria, denominada "Tasy e a construção da Sistematização da Assistência de 
Enfermagem" infere-se sobre as contribuições do Tasy para a realização da SAE e suas influências no ensino deste conteúdo.

\section{Tasy e as contribuições no estabelecimento da relação entre teoria e prática}

A utilização de tecnologias na área da saúde surgiu como uma demanda da denominada Revolução Tecnológica, conforme comentam Pissaia et al. (2018). No entanto, sua efetiva implementação em serviços de saúde iniciou por meio da busca pela qualificação da assistência oferecida à população. Neste sentido, Pissaia e Beschorner (2016) identificam que a presença de tecnologias como o Tasy em serviços da saúde busca alicerçar o ensino e a assistência.

Neste sentido, buscou-se estimular os participantes do estudo a refletirem sobre as contribuições do Tasy no estabelecimento da relação entre teoria e prática. Sobre a temática, E02 relata: "O Tasy ajuda muito, sabe, ele estimula que a gente procure mais as coisas, faça aquilo da sala de aula no próprio computador". Compreende-se que o relato infere sobre a facilidade que o software oferece na realização prática dos conteúdos. Conforme Pissaia et al. (2018), a função da tecnologia na área da saúde é dinamizar os processos de trabalho. Ao dinamizar estes processos, além de qualificar a assistência oferecida ao cliente, a comunicação entre a equipe fica facilitada.

Sobre a facilidade na comunicação entre a equipe, E09 relata: "Foi a primeira vez que mexi no Tasy, ele é muito bom, dá para toda a equipe procurar os dados". Verifica-se que E09 reconhece a importância do Tasy na comunicação entre os membros da equipe. O mesmo participante continua: "Em todos os momentos a equipe acessa, só tem que cuidar pela ética, mas no restante ajuda muito". E09 enfatiza o cuidado com ética na disseminação das informações. No entanto, deixa claros os benefícios da possibilidade de realizar esta troca de informações. Para Pissaia e Beschorner (2016) para que a comunicação interdisciplinar ocorra, não basta um sistema informatizado, mas que os profissionais o abasteçam com as informações do dia a dia assistencial.

Sobre a utilização do Tasy enquanto banco de dados, E07 comenta: "É muito importante trabalhar com o Tasy, na verdade ele ajuda e muito na assistência". Sob o ponto de vista da participante, o software auxilia na qualidade da assistência. o mesmo participante 
complementa: "Ali tem todos os dados, todas as informações que o paciente vai passando durante a vida". A ideia de prontuário eletrônico está presente na visão sobre o Tasy, principalmente em instituições hospitalares conforme comentam Pissaia et al. (2018). Ainda assim, E07 comenta sobre a relação entre teoria e prática: “Achei muito legal fazer isso, na aula a gente aprende a fazer a SAE e depois aqui nós fizemos no sistema com o paciente, acho que aprendi muito mais". A identificação da relação entre teoria e prática demonstra a participação efetiva do participante nas atividades, conseguindo observar as nuances do ensino. Conforme Paurosi et al. (2018), a reflexão sobre o ensino que está ocorrendo em sala de aula espelha o perfil integrador e crítico do estudante.

As identificações sobre a relação entre teoria e prática seguem, podendo ser verificada a de E06, conforme segue: “Eu consegui entender todo o conteúdo". E segue o diálogo: "Na sala de aula, acaba cansando de fazer tudo a mão e tudo é de mentira, aqui não, pra fazer é rapidinho e tem o paciente na frente". Nas considerações de E06 verifica-se que o auxílio do Tasy para a realização da prática facilita a correlação com a teoria. Consonante a isso, Pissaia e Beschorner (2016) reforçam que a presença de sistemas de gestão em saúde qualifica a assistência e o ensino devido à agilidade inerente às práticas.

Ainda sobre isso, E07 comenta: "É muito bom fazer as coisas no Tasy, todo o PE e a SAE dá pra aprender aqui como vai ser feito no serviço". O comentário infere sobre o potencial de ensino que o Tasy desempenha no contexto estudado. Seguindo a conversa, E07 comenta sobre a Sistematização da Assistência de Enfermagem (SAE): "Sabe a SAE, a gente estuda tudo na sala, mas aqui dá pra fazer e ver se funciona com o paciente, pena que não dá para continuar a acompanhar o paciente, mas já da uma ideia". A identificação da SAE, enquanto modelo a ser realizado no Tasy, fortalece a sua função de modelo de gestão em saúde. Para Pissaia et al. (2018), a possibilidade de utilizar softwares de gestão em saúde efetivam o cuidado e possibilitam a implementação de metodologias de trabalho, como no caso da SAE. A relação entre teoria e prática também é fortalecida pela possibilidade de implementar um modelo de gestão efetivo, por meio do software Tasy, conforme inferem Pissaia e Beschorner (2016).

E por fim E02 comenta mais sobre a utilização da SAE no Tasy: “Agora eu consigo fazer a SAE de verdade, na sala a gente fica montando com o livro, mas aqui nós temos a opções e vamos marcando". Nesta fala o participante demonstra a facilidade em realizar a SAE no software. Seguindo a conversa, E02 fala: "Seria interessante ter mais aulas aqui, mais presente do Tasy nas 
aulas sobre o PE e da SAE, pois iríamos apreender com mais facilidade". Identifica assim a necessidade de maior contato com a ferramenta em sala de aula. E finaliza: "Eu já fiz a SAE no Tasy em outros estágios, mas aqui é o mais interessante e certo, porque nos outros lugares era feito por obrigação e aqui a gente faz pelo paciente”. Neste sentido, Tannure e Pinheiro (2014) inferem sobre a importância de tecnologias nos serviços de saúde, auxiliando nos processos de trabalho. Além deste auxílio, Pissaia et al. (2018) indicam a qualificação da assistência oferecida ao cliente como principal modelo de modificação após a implementação de sistemas informatizados.

\section{Tasy e a construção da Sistematização da Assistência de Enfermagem}

Os sistemas informatizados possibilitam a utilização de metodologias de trabalho próprias da saúde. Uma dessas metodologias é a SAE que, conforme Pissaia et al. (2018), é constituída como um modelo integral, holístico e resolutivo de assistência à população. Neste sentido, para Andrade e Silva (2017), a SAE quando implementada em serviços da saúde confere a qualificação do cuidado, dependendo da ação dos profissionais de enfermagem. Ainda assim, Tannure e Pinheiro (2014) indagam sobre a necessidade de buscar alternativas para a implementação da SAE nos serviços da saúde, sendo uma das estratégias o software Tasy, conforme lembram Pissaia e Beschorner (2016).

Sendo assim, torna-se preocupante a forma em que a SAE é ensinada nos cursos de enfermagem. Para Paurosi et al. (2018), o ensino torna-se flexível ao contexto em que o estudante se encontra, principalmente sobre as necessidades da população local. Ainda assim, para Pissaia et al. (2017), o conhecimento sobre a SAE também está atrelado às ferramentas disponíveis para sua implementação nos serviços da saúde.

Com o intuito de acompanhar a realização da SAE no Tasy, abaixo são apresentadas imagens de abas do software que condizem com as etapas do PE realizado com um cliente, denominado "Cliente A" durante a disciplina de Enfermagem na Saúde do Adulto e Idoso II (prática), em campo de estágio. Os clientes descritos abaixo foram acolhidos pela primeira vez no serviço, sendo assim, apresentaremos as etapas de Histórico e Exame físico, Diagnósticos de Enfermagem e Prescrição de Enfermagem. 
Imagem 1. Histórico e exame físico cliente A.

\begin{tabular}{|c|c|c|c|}
\hline \multicolumn{4}{|c|}{ Anamnese/Exame fisico } \\
\hline & Aspecto analisado & Resultado & Observação \\
\hline 1 & Algia & Algia & \\
\hline 2 & Fatores de Risco & Diabético & \\
\hline 3 & Fatores de Risco & Obesidade & \\
\hline 4 & Fatores de Risco & Sedentarismo & \\
\hline 5 & Respiração & Eupnéico & \\
\hline 6 & Batimentos Cardiacos & Normocárdico & \\
\hline 7 & Conhecimento sobre seu problema de saúde & Orientado e pouco confiante & \\
\hline 8 & Temperatura & Normotérmico & \\
\hline 9 & Integridade da Pele & Lesões & \\
\hline 10 & Apetite & Normal & \\
\hline 11 & Estado Nutricional & Obeso & \\
\hline 12 & Via de alimentação & Via oral & \\
\hline 13 & Aspecto Emocionais & Depressivo & \\
\hline
\end{tabular}

Legenda: Aba eletrônica de anamnese/exame físico do software Tasy. Fonte: Autores da pesquisa (2019).

$\mathrm{Na}$ Imagem 1, identifica-se que os participantes realizaram o levantamento das condições de saúde do cliente. Inclui-se o histórico de saúde e doença, além do exame físico, ou seja, os sinais e sintomas presentes no organismo do cliente durante a consulta. Observase que na primeira coluna denominada "Aspecto analisado" são listadas as evidências de situações problema que o cliente apresentou no momento. Já na segunda coluna denominada "Resultados", ficam listados, respectivamente, os fatores relacionados com as evidências indicadas anteriormente. Um exemplo que pode ser extraído, é a evidência de "Integridade da pele", seguida de seu relacionado "Lesões" localizado na segunda coluna. Para Fontana e Pissaia (2018), a identificação de evidências fomenta a capacidade de sintetizar as situações problema do indivíduo, de maneira que as demais etapas do PE a seguirão.

Imagem 2. Diagnósticos de Enfermagem cliente A.

\begin{tabular}{|c|c|c|c|}
\hline & Diagnósticos & Origem Evolução diag & Result esperado \\
\hline 1 & Estilo de Vida Sedentáno & & \\
\hline 2 & Distúrbio na Imagem Corporal & & \\
\hline 3 & Intolerância à Atividade & & \\
\hline 4 & Nutrição Desequilibrada: Mais do que as Necessidades Corporais & & \\
\hline 5 & Ansiedade & & \\
\hline 6 & Baixa Auto estima Crônica & & \\
\hline 7 & Comportamento de Busca de Saúde & & \\
\hline 8 & Desesperança & & \\
\hline 9 & Disposição para Nutrição Melhorada & & \\
\hline 10 & Dor Crônica & & \\
\hline 11 & Dor Aguda & & \\
\hline 12 & Insuficiência do Adulto para Melhorar (o seu Estado de Saúde) & & \\
\hline 13 & Intolerância à Atividade, Risco de & & \\
\hline 14 & Manutenção Ineficaz da Saúde & & \\
\hline
\end{tabular}

Legenda: Aba eletrônica de diagnósticos do software Tasy. Fonte: Autores da pesquisa (2019). 
$\mathrm{Na}$ Imagem 2 estão listados os Diagnósticos de Enfermagem listados pelos participantes durante a aula prática. Verifica-se que os Diagnósticos estão orientados de maneira prioritária, inclusive antecedidos de números ordinais que conferem ênfase a este detalhe. Para este cliente, por exemplo, a prioridade a ser verificada é o Diagnóstico de "Estilo de vida sedentário". Para Tannure e Pinheiro (2014), a identificação de prioridades faz parte da realização efetiva do PE, ao passo que ao elencar prioridades, todo o plano de cuidados será instrumentalizado, buscando-se modificar inicialmente os aspectos prioritários.

Imagem 3. Intervenções de Enfermagem cliente A.

\begin{tabular}{|c|c|c|c|c|c|c|}
\hline \multicolumn{7}{|c|}{ Intervençöes de enfermagem } \\
\hline & Intervenção & Pontos & Seq imp & Código & Origem & Intervalo \\
\hline 1 & Promoção do Exercicio & 9 & & 1 NIC - Classificaçãc 200 & A & \\
\hline 2 & Controle do Peso & 8 & & 1 NIC - Classificaçãc 1260 & A & \\
\hline 3 & Controle da Nutrição & 7 & & 1 NIC - Classificaçãc 1100 & A & \\
\hline 4 & Terapia com Exercicios: Deambulação & 7 & & 1 NIC - Classificaçãc 221 & A & \\
\hline 5 & Aconselhamento & 6 & & 1 NIC - Classificaçãc 5240 & A & \\
\hline 6 & Controle da Dor & 6 & & 1 NIC - Classificaçãc 1400 & A & \\
\hline 7 & Escutar Ativamente & 5 & & 1 NIC - Classificaçãc 4920 & A & \\
\hline 8 & Monitoração de Sinais Vitais & 4 & & 1 NIC - Classificaçãc 6680 & A & \\
\hline 9 & Promoção de Esperança & 4 & & 1 NIC - Classificaçãc 5310 & A & \\
\hline 10 & Redução da Ansiedade & 4 & & 1 NIC - Classificaçã́c 5820 & A & \\
\hline 11 & Terapia com Exercicios: Mobilidade Articular & 4 & & 1 NIC - Classificaçãc 224 & A & \\
\hline 12 & Ensino: Atividade/Exercicio Prescritos & 3 & & 1 NIC - Classificaçãc 5612 & A & \\
\hline 13 & Melhora da Auto-Estima & 3 & & 1 NIC - Classificaçãc 5400 & A & \\
\hline 14 & Aconselhamento Nutricional & 2 & & 1 NIC - Classificaçãc 5246 & A & \\
\hline 15 & Alimentação & 2 & & 1 NIC - Classificaçãc 1050 & A & \\
\hline 16 & Assistência no Autocuidado: Alimentação & 2 & & 1 NIC - Classificaçãc 1803 & A & \\
\hline 17 & Assistência para Reduzir o Peso & 2 & & 1 NIC - Classificaçãc 1280 & A & \\
\hline 18 & Encaminhamento & 2 & & 1 NIC - Classificaçã́c 8100 & A & \\
\hline 19 & Identificação de Risco & 2 & & 1 NIC - Classificaçãc 6610 & A & \\
\hline 20 & Terapia Nutricional & 2 & & 1 NIC - Classificaçãc 1120 & A & \\
\hline 21 & Controle da Hiperglicemia & 1 & & 1 NIC - Classificaçãc 2120 & A & \\
\hline 22 & Controle da Hipoglicemia & 1 & & 1 NIC - Classificaçã́c 2130 & A & \\
\hline 23 & Cuidados com Lesões & 1 & & 1 NIC - Classificaçãc 3660 & A & \\
\hline
\end{tabular}

Legenda: Aba eletrônica de intervenções de enfermagem do software Tasy. Fonte: Autores da pesquisa (2019).

$\mathrm{Na}$ Imagem 3 estão descritas as Intervenções de Enfermagem realizadas pelos participantes. Identifica-se que as intervenções também aparecem por ordem de prioridade e ligadas às linhas em que os Diagnósticos de Enfermagem aparecem na Imagem 2. Utiliza-se como exemplo o Diagnóstico de Enfermagem de "Estilo de vida sedentário" localizado na linha "1", eleito como prioridade na sua aba é ligada a Intervenção de Enfermagem "Promoção do exercício" também localizado na linha "1" da respectiva aba do software Tasy. Para Fontana e Pissaia (2018), a construção das Intervenções de Enfermagem está ligada ao centro do plano de cuidados. Em suma, representa toda a assistência que a equipe de enfermagem se propõe a realizar para o cliente. 
Neste sentido, com as imagens destacadas percebe-se que o Tasy representa a potencialidade de realização da SAE de maneira que complementa a realização de maneira manual durante a teoria. Para Pissaia e Beschorner (2016), as interações dos estudantes com o software dinamizam a compreensão sobre o PE e o saber para a realização da SAE, além de possibilitar a comunicação integrada entre as equipes de trabalho. Ainda assim, Pissaia et al. (2018) instrumentalizam que a presença de sistemas informatizados qualificam a assistência oferecida ao cliente, principalmente na oferta de resolutividade nas situações problema.

\section{CONSIDERAÇÕES FINAIS}

Durante a análise das contribuições do software Tasy para o estabelecimento da relação entre teoria e prática, percebeu-se que os participantes o descrevem como facilitador. Surgiram também indicações de sua importância como registro das informações assistenciais e, por consequente, qualificador do serviço de saúde. No entanto, as discussões permaneceram atreladas a sua potencialidade na construção de um ensino qualificado ao possibilitar o vislumbre de um conteúdo em diferentes cenários, teórico e prático.

Ainda sobre a relação entre teoria e prática, identifica-se que os participantes reconhecem a necessidade de presenciarem a utilização do software, visto a facilidade e dinamismo das práticas assistenciais que estão atreladas a ele. Aliados aos relatos verificouse que os participantes tiveram a oportunidade de realizar a SAE com um cliente atendido no serviço campo de estágio.

Sendo assim, considera-se que software Tasy contribui para o estabelecimento da relação entre teoria e prática, principalmente ao possibilitar vivências tecnológicas e diferenciadas aos participantes. Aliado a isso, a experiência qualifica a realização do ensino da SAE, o manuseio de sistemas de gestão em saúde e tecnologias necessárias ao trabalho do enfermeiro contemporâneo. 


\section{REFERÊNCIAS}

ANDRADE, J. S.; SILVA, F. J. C. P. Diretrizes para a sistematização da assistência de enfermagem hospitalar. In: Congresso Internacional de Enfermagem, 2017.

BARDIN, L. Análise de Conteúdo. Tradução: Luís Augusto Pinheiro. São Paulo: Edições 70, 2016.

BRASIL. Conselho Nacional de Saúde. Ministério da Saúde (BR). Portaria 466/2012. Brasília (DF), 2012.

FONTANA, M.; PISSAIA, L. F. O uso do processo de enfermagem como ferramenta de apoio para o cuidado da criança na atenção domiciliar. Research, Society and Development, v. 7, n. 11, p. 1371-1576, 2018.

MOREIRA, M. A. Metodologias de pesquisa em ensino. $1^{\text {a }}$ Ed. São Paulo. Livraria da Física, 2011.

PAUROSI, D. R.; ASCARI, R. A.; SILVA, O. M.; ASCARI, T. M. Diretrizes operacionais para uma central de Material e esterilização odontológica: Uma proposta da enfermagem. Revista UNINGÁ, v. 17, n. 2, 2018.

PHILIPS. [internet] Disponível em: https://www.philips.com.br/healthcare/product/HCNOCTN306/tasy. Acesso em: 25 fev. 2018.

PISSAIA, L. F.; COSTA, A. E. K.; MORESCHI, C.; REMPEL, C.; CARRENO, I.; GRANADA, D. Impacto de tecnologias na implementação da sistematização da assistência de enfermagem hospitalar: uma revisão integrativa. Revista de Epidemiologia e Controle de Infecção, v. 1, p. 1-20, 2018.

PISSAIA, L. F.; COSTA, A. E. K.; MORESCHI, C.; REMPEL, C. Tecnologias da informação e comunicação na assistência de enfermagem hospitalar. Revista de Epidemiologia e Controle de Infecção, v. 7, p. 1-10, 2017.

PISSAIA, L. F.; BESCHORNER, C. E. Implantação de um ambulatório de média complexidade no Vale do Taquari/ RS: um relato de experiência. Cinergis, Santa Cruz do Sul, v. 17, n. 4, p. 307-312, out./dez. 2016.

SILVA, S. B.; GONÇALVES, N. S.; SANTOS, D. C. Implantação de um modelo de descentralização de auditoria de contas hospitalares em um hospital de grande porte na região sul do Brasil. Revista de Administração em Saúde, v. 17, n. 69, 2017.

TANNURE, M. C.; PINHEIRO, A. M. SAE: Sistematização da Assistência de Enfermagem: Guia Prático. Rio de Janeiro, Guanabara Koogan, 2014.

YAMAMOTO, T. T. I.; BANDIERA-PAIVA, P.; ITO, M. Avaliação da usabilidade de interface gráfica de dois sistemas de gestão hospitalar. Journal of Health Informatics, v. 7, n. 2, 2015.

\section{(cc) Br}

Este trabalho está licenciado com uma Licença Creative Commons - Atribuição 4.0 Internacional. 